Preprint typeset using $\mathrm{LAT}_{\mathrm{E}} \mathrm{X}$ style emulateapj v. 08/22/09

\title{
MILGROM RELATION MODELS FOR SPIRAL GALAXIES FROM TWO-DIMENSIONAL VELOCITY MAPS
}

ERIC I. BARNES

Department of Physics, University of Wisconsin-La Crosse, La Crosse, WI 54601

Arthur Kosowsky

Department of Physics and Astronomy, University of Pittsburgh, Pittsburgh, PA 15260

\author{
J.A. SEllWood
}

Department of Physics and Astronomy, Rutgers University, Piscataway, NJ 08854

\begin{abstract}
Using two-dimensional velocity maps and I-band photometry, we have created mass models of 40 spiral galaxies using the Milgrom relation (the basis of modified Newtonian dynamics, or MOND) to complement previous work. A Bayesian technique is employed to compare several different dark matter halo models to Milgrom and Newtonian models. Pseudo-isothermal dark matter halos provide the best statistical fits to the data in a majority of cases, while the Milgrom relation generally provides good fits as well. We also find that Milgrom models give mass-to-light ratios that roughly correlate with galaxy color, as predicted by stellar population models. A subsample of galaxies in the Hydra cluster follow a tight relation between mass-to-light and color, but one that is significantly different from relations found in previous studies. Ruling out the Milgrom relation with rotational kinematics is difficult due to systematic uncertainties in the observations as well as underlying model assumptions. We discuss in detail two galaxies for which the Milgrom relation appears to fail and find that relaxing the assumption of constant stellar mass-to-light ratio can maintain Milgrom models' viability.

Subject headings: galaxies: fundamental parameters - galaxies: kinematics and dynamics — galaxies: dark matter — galaxies: stellar content
\end{abstract}

\section{INTRODUCTION}

The vast majority of spiral galaxy rotation curves are flatter than Newtonian predictions at large distances from galactic centers (Salpeter 1978; Rubin 1979; Sancisi \& Allen 1979; Krumm \& Salpeter 1979; Sancisi et al. 1979; van Moorsel 1982; Rohlfs et al. 1986). Galaxies that do not show this behavior often show peculiar morphologies or perturbing interactions. This asymptotic flatness of rotation curves, which is not perfect (for a more in-depth discussion of this point see, e.g., Persic et al. 1996; Catinella et al. 2006), disagrees with the Newtonian prediction in which the circular orbital speed due to the gravity from a galaxy's luminous material should decrease at distances which enclose substantial fractions of a galaxy's visible mass. This type of discrepancy is usually viewed as evidence for a nonluminous mass component, known as a dark matter halo. This picture of luminous matter embedded in dark matter halos has become the paradigm of galactic structure, and recent work involving galaxy clusters (Clowe et al. 2006; Bradac et al. 2006) has presented strong evidence that this accurately reflects astrophysical reality. Since a precise mass distribution of the visible matter is difficult to determine directly, the dark matter distribution is also not known. Previous studies (e.g., Blais-Ouellette et al. 2001; Barnes et al. 2004, hereafter BSK) have found that the choice of dark matter distribution significantly impacts the derived mass of the luminous material.

Electronic address: barnes.eric@uwlax.edu

Electronic address: kosowsky@pitt.edu

Electronic address: sellwood@physics.rutgers.edu
A different phenomenological approach to rotation curve fitting is provided by the fitting formula originally proposed by Milgrom (Milgrom 1983a,b, d) which he termed MOND, for "modified Newtonian dynamics." The original goal of MOND was to use only the luminous matter to explain two widely observed properties of spiral galaxies; asymptotic flatness of galactic rotation curves and the well-known Tully-Fisher relation $L \simeq v^{\beta}$ (Tully \& Fisher 1977). A tight relation between a galaxy's luminosity $L$ and the asymptotic value of the galaxy's circular speed $v$ appears to be valid for all varieties of spirals, high and low surface brightness as well as barred and unbarred galaxies (Spravberry et al. 1995;: Zwaan et al. 1995; Courteau \& Rix 1999; Courteau 2003). McGaugh et al. (2000) has convincingly argued that luminosity is actually a stand-in for total baryonic mass; i.e., the same Tully-Fisher relation holds for both low and high surface brightness spirals if baryonic mass (stars and gas) is used in place of luminosity. When this baryonic Tully-Fisher relation is examined, $M_{\text {baryonic }} \propto v^{4}$ (McGaugh 2005). These observations are built into the MOND formalism as follows. The total acceleration due to a gravitating object is given by the expression

$$
\mathbf{a} \mu\left(a / a_{0}\right)=\mathbf{a}_{N},
$$

which relates the total acceleration a to the Newtonian acceleration $\mathbf{a}_{N}$ through a function $\mu$ satisfying the limiting forms $\mu \rightarrow 1$ when $a \gg a_{0}$ and $\mu \rightarrow a / a_{0}$ when $a \ll a_{0}$ (Milgrom 1983a). The new universal constant $a_{0}=1.2 \times 10^{-10} \mathrm{~km} / \mathrm{s}^{2}$ is obtained from fits to measured rotation curves and is the same for all galaxies. 
The exact form of the interpolating function is essentially unconstrained by rotation curve data; throughout this paper we use $\mu(x)=x / \sqrt{1+x^{2}}$ (Begeman et al. 1991). It has long been realized that Equation (11) violates conservation of linear momentum; a more acceptable gravitational modification alters the Poisson equation for the gravitational potential $\phi$ (Bekenstein \& Milgrom 1984),

$$
\left.\nabla \cdot\left[\mu\left(\frac{a}{a_{0}}\right) \nabla \phi\right)\right] .
$$

This modification of the gravitational field explicitly conserves energy and momentum, as it is based on a Lagrangian formulation. However, in the limited case of spiral galaxies like those considered here, the force law modification of Equation (1) is a sufficiently accurate approximation.

In the inner regions of typical spiral galaxies, the characteristic acceleration scale $a \gg a_{0}$ and the gravitational force reduces to the usual Newtonian force. In the outer regions of spiral galaxies, $a \ll a_{0}$ (the so-called MOND regime) leading to the acceleration having a $1 / r$ dependence, asymptotically flat rotation curves, and an enclosed mass varying like the flat rotation speed to the fourth power. Assuming a constant mass-to-light ratio $\Upsilon$ results in the Tully-Fisher relation. A number of previous studies have utilized Equation (11) to describe rotation curves (Kent 1987; Begeman et al. 1991; Sanders 1996; Sanders \& Verheijen 1998; de Blok \& McGaugh 1998; Gentile et al. 2004). Their success in accurately modeling rotation curves with one free parameter (two if a bulge is present) rivals dark matter halo models that require at least two additional parameters. The empirical accomplishments suggest that regardless of the veracity of the hypothesis that Newtonian dynamics is modified, Equation 1 (the Milgrom relation) is a powerful and compact method for characterizing the rotational kinematics of spiral galaxies.

In this work, we regard the Milgrom relation as an empirical fitting formula for rotation curve data. In particular, we make no assumptions about properties of dark matter halos, or modifications to gravitation, from which the Milgrom relation might arise. But as emphasized in McGaugh (2004), Equation (1) is the effective force law for the visible matter in spiral galaxies, regardless of whether the force comes from a distribution of dark matter or from MOND, which seems increasingly unlikely in the face of evidence like that presented in Clowe et al. (2006); Bradac et al. (2006) (but see also Angus et al. 2006). This paper remains agnostic about the ontological status of the Milgrom force law. However, we note that if galactic dark matter is taken as a certainty, then the success of the Milgrom relation in predicting spiral galaxy rotation curves reflects an underlying dynamical process in galaxy formation which is not well understood and is deserving of further scrutiny. In this work, we are interested in determining i) the extent to which this dynamical relation holds and ii) specific situations in which the relation might not hold.

This paper performs Milgrom fits to the same sample of spiral galaxies considered in BSK, and provides a statistical comparison with the dark matter halo fits. The halo profiles considered in BSK are the familiar Navarro et al.
(1996, hereafter NFW)

$$
\rho_{\mathrm{NFW}}(r)=\rho_{s} \frac{r_{s}^{3}}{r\left(r+r_{s}\right)^{2}},
$$

where $\rho_{s}$ is a characteristic density and $r_{s}$ is a scale length; pseudo-isothermal (PI)

$$
\rho_{\mathrm{PI}}=\frac{\rho_{0}}{1+\left(r / r_{c}\right)^{2}},
$$

with central density $\rho_{0}$ and core radius $r_{c}$; and a simple power law

$$
\rho(r)=\rho_{0}\left(\frac{r}{r_{l}}\right)^{\alpha},
$$

along with a Newtonian stars-only fit which is useful for comparing with both Milgrom and dark halo fits.

Sample selection, photometric and kinematic data, and modeling procedures are discussed in $\S 2$. Along with the results of Milgrom fits to this data $(\S 3.1$ ), we obtain the $\Upsilon$-color relation for these models $(\S[3.2)$. This sample of galaxies contains 8 Hydra cluster galaxies, and we focus on these galaxies to investigate the claim that Milgrom models produce $\Upsilon$ values that closely agree with population synthesis predictions and therefore provide likely estimates of luminous mass. We also compare the Milgrom model fits to the dark halo fits from BSK, utilizing a Bayesian technique that quantitatively compares models with different numbers of free parameters. A description of this technique along with model comparisons is given in Section 4. Section [5] discusses the impact of systematic distance uncertainties. Finally, we discuss two anomalous galaxies in $\S[$ and consider the validity of these statistical goodness-of-fit measures in the presence of known systematic errors in our underlying assumptions. Color gradients within individual galaxies and unobserved gas point to small variations in their mass-light ratios, at a level which naturally explains Milgrom fit discrepancies.

\section{DATA \& MODELING}

BSK contains a detailed discussion of the data and fitting methods used in this analysis, and the interested reader is pointed there for a detailed description. Briefly, we use a sample of 40 high surface brightness galaxies with $I$-band images and Fabry-Perot two-dimensional velocity maps. These data have been provided by Povilas Palunas and Ted Williams and a thorough description of the acquistion and reduction of the data can be found in Palunas \& Williams (2000). We decompose the $I$ band images into disk and bulge components when necessary. The disk is assumed to be radially symmetric and infinitesimally thin and the bulge is assumed to be spheroidal.

A mass model is determined once the mass-light ratios for the disk $\left(\Upsilon_{D, I}\right)$ and the bulge $\left(\Upsilon_{B, I}\right)$ are specified. Despite evidence for gradients in $\Upsilon$ factors $(e . g .$, de Jong 1996), we first consider $\Upsilon$ values to be spatially constant; we relax this assumption in $\S 6.3$. The $I$ subscript indicates mass-light ratios determined from $I$-band photometry. We neglect any contribution from gas, as we have no information about the radial distribution of gas in the galaxies in our sample; however, the gas in late-type galaxies like those in our sample is likely to contribute 
$\leq 10 \%$ of the circular speed at any radius (Broeils 1992; Verheijen 1997). Neglecting it will cause a slight systematic overestimate of $\Upsilon_{D, I}$ and can lead to models mildly underpredicting circular speeds in the outer parts of galaxies. It could be argued that omitting the gas contribution will cause our stars-only Milgrom models to describe the data worse than other models simply because we are not including all relevant information; i.e., our Milgrom models are born at a disadvantage. However, our Milgrom models do not show any systematic trends indicating that large amounts of gas would improve the models; the Milgrom models do not systematically underpredict the rotation speeds at the edge of the kinematic data. We discuss the impact of this omission in more detail for two interesting galaxies in $\S$.

The disk-bulge mass model determines the Newtonian circular speed $v_{c}(\theta)$ as a function of angular separation from the center of the galaxy. This is converted to a circular speed $v_{c}(r)$ as a function of distance from the center of the galaxy, using the angular diameter distance to the galaxy given by the Hubble distance (systematic errors associated with this simple distance assumption are discussed in Sec. [5.1). Equation 10 converts this Newtonian circular velocity to a Milgrom-relation circular velocity. The Newtonian disk-bulge mass model is also combined with the standard dark matter halo models (Eqs. 3 4 and 5) to give the total rotation curves in these cases.

The rotation curve inferred from the model mass distribution is compared with the actual galaxy rotation as measured by the Fabry-Perot maps. Throughout this paper, we fix the position angle $\phi_{0}$, inclination angle $i$, and systemic velocity $v_{s}$ of each galaxy at the values deduced from earlier fits of axisymmetric flow patterns to our kinematic data (Barnes \& Sellwood 2003). We have found the values of these parameters that minimize $\chi^{2}$ between the two-dimensional velocity map and a planar axisymmetric flow pattern. The fit therefore yields a set of mean orbital speeds, and their uncertainties, at equally-spaced radii which we show as the "data" in subsequent figures illustrating rotation curves. Since we fit axisymmetric mass models, we could, in principle, fit only this one-dimentional rotation curve, which represents the speed averaged in annular bins, provided the weights are computed correctly. However, the full twodimensional maps have several important advantages, including clear identification of outlying pixels and identification of non-axisymmetric velocity patterns (usually bars or spirals) which cause systematic errors in rotation curves obtained from one-dimensional velocity measurements. Of course, identifying non-axisymmetric patterns is only half the battle. We are looking towards collecting data for a sample chosen with minimal spiral and/or bar structures that will involve fewer systematic effects and will allow better model discrimination.

\section{MILGROM FITS}

The mass model incorporating disk and bulge components gives a Newtonian circular velocity $v_{c}(r)$ and acceleration $a_{N}(r)$. Equation 1 then gives a total acceleration $a(r)$; equating these accelerations with centripetal accelerations give us the Milgrom circular speeds. Note that for the galaxies considered here, the simple acceleration approximation in Eq. 1 gives essentially the same answer as the physically more realistic Poisson-like Eq. 2 which is numerically more challenging to solve. Using previously determined projection angles and systemic velocities (Barnes \& Sellwood 2003), which are held fixed, we project a one-dimensional speed profile into a twodimensional velocity map. This model velocity map is compared to the data velocity map, pixel by pixel.

A small number of pixels exhibit what are clearly anomalous inferred velocities; these are almost all isolated pixels in outer regions of galaxies, where the velocity is much different than the smooth velocity field defined by the surrounding pixels. These points are due to imperfect filtering of cosmic rays and unmodelled line emission in the data analysis pipeline, which spuriously fits the pixels as having high velocity and low dispersion. To reduce the influence of these non-normal errors, we find the best-fit values for $\Upsilon_{D, I}$ and $\Upsilon_{B, I}$ by minimizing the Tukey biweight parameter (Press et al. 1992),

$$
\chi_{b}^{2}=\frac{1}{N} \sum_{i=1}^{N} \begin{cases}z_{i}^{2}-\frac{z_{i}^{4}}{c^{2}}+\frac{z_{i}^{6}}{3 c^{4}} & \left|z_{i}\right|<c, \\ \frac{c^{2}}{3} & \text { otherwise }\end{cases}
$$

where $z_{i} \equiv\left(D_{i}-M_{i}\right) / \sigma_{i}, D_{i}$ and $M_{i}$ are the respective measured and mass-model velocities in pixel $i, \sigma_{i}$ is the measurement standard error on $D_{i}, c$ is a constant, and $N$ is the number of data points. Note that in the limit $c \rightarrow \infty$, this reduces to the usual expression for reduced chi-squared $\chi_{r}^{2}=1 / N \sum_{i=1}^{N}\left(D_{i}-M_{i}\right)^{2} / \sigma_{i}^{2}$. While data values that differ from the model prediction by $\lesssim c \sigma_{i} / 2$ contribute to $\chi_{b}^{2}$ almost exactly as for the conventional $\chi_{r}^{2}$, the contribution from data values that are farther from the model prediction by $c \sigma_{i}$ does not change as the parameters are adjusted, and outlying data therefore do not influence the resulting parameter values. Press et al. (1992) recommend $c=6$, but non-circular flow patterns in our galaxies suggest that a larger value would be more appropriate; after some experimentation we find $c=10$ eliminates the influence of the extreme outlying velocities while allowing the great majority of the pixels to contribute to the fit with almost full weight. Only a handful of pixels per galaxy image are ignored using this procedure.

\subsection{Parameter Values and Rotation Curves}

The best-fit $\chi_{b}^{2}, \Upsilon_{D, I}$ and $\Upsilon_{B, I}$ parameters, statistical uncertainties $(\sigma)$, and systematic uncertainties $(s)$ for the Milgrom mass models are given in Table 1. We also include the ratio of the distance to the edge of the kinematic data $R_{\mathrm{k}, \max }$ to the disk scalelength $r_{d}$ for each galaxy. On average, the kinematic data extends to $\approx 4.5 r_{d}$ and covers the visible disk. The uncertainty calculations are discussed in the next paragraph. The average $\Upsilon_{D, I}$ is 1.9 and the average $\Upsilon_{B, I}$ is 2.9 , with wide variation in both values. Generally, the Milgrom model values are lower than the Newtonian stars-only values; the ratio of $\Upsilon$ between the Milgrom models described here and the Newtonian stars-only models of BSK are 0.6 and 0.5 for the disk and bulge components, respectively.

Statistical uncertainties have been calculated using Markov Chain Monte Carlo (MCMC) techniques (e.g., Christensen et al. 2001; Kosowsky et al. 2002) to map out allowed regions of parameter space. Projections of the boundary which encloses the most likely $68 \%$ of the 
models then provide the statistical uncertainties. The average statistical uncertainties are 0.08 for $\Upsilon_{D, I}$ and 0.3 for $\Upsilon_{B, I}$. The quoted systematic uncertainties in the fitted $\Upsilon_{I}$ values are due to the uncertainties in the galaxy's inclination and position angle. A previous paper (Barnes \& Sellwood 2003) estimated systematic uncertainties in inclination and position angles caused by spirals and other nonaxisymmetric structure in the galaxies. The best-fit value plus the estimated high and low limits for each of the two projection angles yield nine different combinations of inclination and position angle which give nine separate estimates for $\Upsilon_{I}$ values. We take the systematic uncertainty in a $\Upsilon_{I}$ value to be half the range of these nine $\Upsilon_{I}$ values. As in BSK, the systematic uncertainties dwarf the statistical ones with an average $s \Upsilon_{D, I}$ of 0.5 and an average $s \Upsilon_{B, I}$ of 0.8 .

Figure 1 illustrates the rotation curves of six galaxies (assuming fixed Hubble distances). The best-fit Milgrom models (solid lines) are shown along with the data points derived from velocity maps. For comparison, we also show the PI halo models (dot-dashed lines) from BSK. The top two panels are for galaxies in which the Milgrom fit is favored over the various BSK halo models. The middle set of panels show fits to galaxies with nonaxisymmetric structure in which the Milgrom fit is less favored than the other BSK models. The bottom panels show curves for axisymmetric galaxies in which the Milgrom fit is less favored than the BSK models. These galaxies will be discussed in more detail in $\S 6$. One of the most striking things about this figure is the close similarity bewteen the Milgrom and halo curves; the differences in circular speeds between the models shown are rarely much larger than $10 \mathrm{~km} / \mathrm{s}$. Large differences in likelihoods between the models are due to small variations in model velocities over very large numbers of pixels in the two-dimensional velocity maps.

\subsection{Color- $\Upsilon$ relations}

Stellar population synthesis models predict correlations between galaxy color and $\Upsilon_{D, I}$ value, with red galaxies having larger $\Upsilon_{D, I}$ values than blue galaxies (e.g., Bell \& de Jong 2001; Bell et al. 2003). As in BSK, we have created galaxy colors obtained from the ESOLV aperture magnitudes, as recorded in the NASA/IPAC Extragalactic Database (NED). In principle, we should compare the $\Upsilon_{D, I}$ with the color of the disk alone, but NED gives magnitudes of the disk and bulge combined. The bulge light in our mostly late-type galaxies is a small fraction, typically $\leq 10 \%$, of the total in the $I$-band, so any correction for the bulge to the total color is likely to be small. We have corrected the NED magnitudes for galactic extinction using values from Schlegel et al. (1998) and for internal extinction using the prescriptions of Tully et al. (1998). We estimate errors in our colors to be around $0.15 \mathrm{mag}$.

In BSK, we found that the model $\Upsilon$ values for the galaxies in this sample did not have any trend, and instead follow a flat distribution, albeit with large scatter. We present here the color- $\Upsilon_{D, I}$ relation for the Milgrom models for these same galaxies. Figure 2 shows the $\Upsilon_{D, I}$ value of each galaxy plotted versus its dereddened color for Newtonian stars-only models (top frame) and Milgrom models (bottom frame). The error bars reflect the statistical and systematic uncertainties added in quadra- ture. Galaxies marked by squares have no bulge components. The solid lines in each frame represent the correlation given in Bell et al. (2003). The dashed lines are the best-fit linear fits to the data. Contrary to the findings of BSK, we see a trend in the Milgrom color- $\Upsilon$ plane similar to that expected by stellar population models. The slopes of these lines show some discrepancy but there is still a large scatter in our points.

This sample of 40 galaxies contains 8 which belong to the Hydra cluster. The color- $\Upsilon_{D, I}$ plot of these galaxies is shown in Figure 3 As before, the Bell et al. (2003) correlation is shown as a solid line, while the best linear fit to the data (marked by diamonds) is the dashed line. This linear fit appears to accurately describe the correlation present in the data with little scatter. The large offset between the two lines is surprising since McGaugh (2004) found that MOND $\Upsilon_{D}$ values matched the Bell et al. (2003) line remarkably well. There are a number of possible explanations of the discrepancy between the Hydra galaxies and the Bell et al. (2003) correlation. One possibility is that the offset is due to errors in the adopted distances to these galaxies (see $\S 5$ for a detailed discussion of the impact of distance on MOND $\Upsilon$ values). However, Hudson et al. (1997) give a Fundamental Planebased, Malmquist bias corrected distance to the Hydra cluster of $52.6 \pm 2.5 \mathrm{Mpc}$ and the galaxies in this plot have adopted distances that average to $52.6 \pm 4.2 \mathrm{Mpc}$. Metallicities and initial mass functions of galaxies have also been shown to strongly impact the slope and intercept of the color- $\Upsilon_{D, I}$ correlation (Bell \& de Jong 2001); these possibilities would require further observations to explore in more detail.

\section{MODEL COMPARISONS}

Previous studies have rarely attempted direct comparisons between Milgrom fits and halo model fits. Here we compare the Bayesian probabilities of halo models to Milgrom models, taking into account the differing number of free parameters in the models. A brief summary of the necessary Bayesian probability theory is given in the Appendix. The model comparisons here are based on statistical fits only. Evaluation of some systematic sources of error and their potential impact on straightforward statistical model comparison are considered in the following sections.

We are considering several different models with different numbers of parameters and we would like to know which provides the most economical description of the data. Specifically, we have compared our Milgrom fits to various halo fits (PI, NFW, and power law halos), along with Newtonian stars-only fits for illustration (for a full discussion of the various halo models, see BSK). We have used the following parameter ranges for (uniform) prior probability values: (PI) $\Delta \rho_{0}=2 \mathrm{M}_{\odot} / p c^{3}, \Delta r_{c}=20$ $\mathrm{kpc} ;(\mathrm{NFW}) \Delta c=20, \Delta v_{200}=300 \mathrm{~km} / \mathrm{s} ;$ (power law) $\Delta v_{0}=300 \mathrm{~km} / \mathrm{s}, \Delta \alpha=2$. Altering the priors will change a given model's probability, but other sensible choices do not change our basic results. Table 2 lists the logarithms of the probability ratios $\lambda$ of the BSK models to Milgrom fits. $\lambda$ values $<0$ indicate that the Milgrom model is preferred. The large ratios between some halo and Milgrom models are due to the large numbers of data points in the two-dimensional velocity data, resulting in very small statistical error bars and large likelihood ratios between 
models with similar rotation curves. A simpler model comparison test based on Bayesian information criteria (BIC) (Liddle 2004) agrees with the results we present here.

Surprisingly, the Newtonian stars-only model is strongly favored over Milgrom for nine galaxies, and the models are comparable for two others (ESO $317 \mathrm{~g} 41$ and $445 \mathrm{~g} 39)$. In fact, the Newtonian stars-only model is preferred over all other models for ESO 438g08 and 382g06. Of these 11 galaxies, 5 have substantial bars, 3 have noticeable spirals, and 1 has a close, possibly interacting, companion (ESO 438g08). Since it is possible that these circumstances introduce a bias into the model comparison, we do not focus our attention on these galaxies. The two remaining galaxies (ESO 323g42 and 382g06) appear axisymmetric (no large bars or strong spiral structure). These galaxies are discussed in detail in $\S$ 6

For three galaxies, Milgrom models are preferred or are comparable to all other models: ESO 322g76, ESO 445g15, and ESO 501g68. ESO 445g15 and ESO 501g68 have bars while ESO $322 \mathrm{~g} 76$ has moderately strong spiral structure. All 3 have velocity maps that extend at least 2.5 disk scalelengths and their rotation curves have flattened by the last data point. Of the 40 galaxies analyzed, these are the only galaxies for which Milgrom is preferred over the PI halos. Milgrom models are preferred over NFW halos in 7 cases. These results suggest that, in general, the simplicity (fewer free parameters) of Milgrom models does not statistically outweigh the ability of halo models to provide better fits.

Due to its poor performance relative to the halo models, it is tempting to conclude immediately that the Milgrom relation describes galaxy rotation curves only by accident, undermining the strongest reason for considering MOND as a modification of gravity. However, this conclusion would be premature without detailed consideration of systematic uncertainties. It is dfficult to find cases where the poorer fit of the Milgrom model cannot be accounted for by reasonable known systematic errors.

Milgrom models are highly constrained, and we have made several implicit assumptions which have substantial effects on the rotation curves: 1) all stellar motions are circular; 2) the visible stellar light accounts for all of the mass in the galactic disk (neglecting, in particular, any gas, which is known to be non-negligible in some galaxies); 3) the mass-light ratio is constant throughout the galaxy; 4) the adopted inclination angle is correct; and 5) the galaxy distance is given by the Hubble distance. Not one of these assumptions are exactly true, and in many galaxies these assumptions are known to be incorrect at levels which would change the rotation curve by an amount significantly larger than the statistical errors. The sample considered here, in particular, was chosen to probe large-scale velocities in the local universe, not because of its particular suitability for galaxy dynamics; we have analyzed it because of its large sample of high-quality two-dimensional velocity measurements. Milgrom models have essentially no ability to compensate for any failure of these assumptions as they are determined completely by the distribution of visible matter. Halo models, by contrast, have two degrees of freedom which are unconnected to the distribution of visible matter and otherwise unconstrained by direct observation, and can therefore compensate to some degree for any in- correct assumptions about the visible matter. In other words, beyond the fact that halo models will generically provide better fits because they have more parameters, they are also more flexible in compensating for systematic errors, which is not reflected in purely statistical comparisons.

In the remainder of this paper, we consider in more detail some of the important systematic errors which might affect the analysis presented here. We then focus on two sample cases for which Newtonian fits are statistically superior to either Milgrom or dark halo fits, and discuss which possible explanations for the discrepancy are reasonable.

\section{SYSTEMATIC EFFECTS}

\subsection{Distances}

A substantial source of uncertainty is the adopted distance to a galaxy. $\Upsilon$ values are inversely proportional to distance in both Newtonian and Milgrom mass models. Unfortunately, a significant fraction $(\approx 50 \%)$ of the galaxies in this sample were originally observed to study peculiar velocities in the region toward the "Great Attractor" and may therefore have larger-than-usual peculiar velocities and distance uncertainties. Bothun et al. (1992) used the Tully-Fisher relation to estimate distances to this subsample of galaxies, finding differences between redshift distance and Tully-Fisher distance of typically about $25 \%$. While for Newtonian (stars-only and halo) fits this simply introduces a scale uncertainty in $\Upsilon$ values, Milgrom fits are subject to a further distance dependence, since they depend on the dimensional parameter $a_{0}$. Changing the distance to a galaxy changes the acceleration scale at a given angular separation from the galaxy center, and thus changes the inferred radius at which the non-Newtonian behavior sets in. For example, suppose the distance to a galaxy has been adopted at half its actual distance. Given angular separations now correspond to physical distances that are half the actual values. Since the accelerations are inversely related to physical distances, the accelerations increase. These increases then push the location where acceleration becomes comparable to $a_{0}$ farther out in the disk. Moving a galaxy closer forces the Milgrom fit to be closer to Newtonian, while adopting a distance larger than the actual value increases the discrepancy between Milgrom and Newtonian fits.

While Milgrom fits are fairly sensitive to the adopted distance scale, this fact can be exploited as a method for determining distances. As related in Sanders \& McGaugh (2002), changing the adopted distance to a galaxy until a satisfactory Milgrom fit is obtained gives an independent distance measurement that is often in accordance with Tully-Fisher distances (which is not surprising, as the Tully-Fisher relation is implied by the Milgrom relation). We have investigated this possibility by including the distance to galaxies as another free parameter in Milgrom fits. For our data set, most fits of this kind are unstable, leading to models with galaxies unreasonably nearby or far away. Part of the reason for this behavior is due to bars and spirals. Nonaxisymmetric features tend to lower rotational velocities, making axisymmetric models overshoot the data, especially in central regions. In such a case, the fitting routine 
can better match the data by moving the galaxy farther away, thereby lowering the $\Upsilon$ values, making the baryonic component insignificant, and allowing the nonNewtonian MOND behavior to dominate. This is similar to the behavior of some galaxies that are best fit with a halo alone, as noted in BSK. One of our galaxies (ESO 438g08) has a declining rotation curve that is best fit by the Newtonian stars-only model and returns a terrible fixed-distance MOND fit. Once the distance is allowed to change, the galaxy is moved unbelieveably closer so that the fit can become more Newtonian. If this galaxy's kinematics can be shown to be due only to the matter within the galaxy, it would pose quite a problem for MOND, as well as for any commonly considered dark matter halo. However, the galaxy has a nearby, possibly interacting, companion; while we cannot definitively say that tidal interaction is causing the declining rotation curve, neither can we rule it out.

In an attempt to remove the effects of aggravating central asymmetries, we have excised pixels from the centers of the galaxy images and re-fit the remaining data with a Milgrom model that has the distance as an additional free parameter. If the free parameter distance varies by more than a factor of 3 from the Hubble distance we used in the fixed distance fitting, we consider the distance fit unstable. We fit each galaxy 10 times, each time increasing the number of excised pixels. The first attempt utilizes all the pixels and each successive trial removes an additional $5 \%$ of the total radial data range from the central regions. Fifteen galaxies cannot find acceptable distance fits for any amount of excised pixels. Fourteen galaxies have acceptable fits using all the data. Twenty-two galaxies have at least three consecutive fits with acceptable distances; however, the distances that these fits return never agree with each other to better than $30 \%$ of the Hubble distance. So while the adopted Hubble distance necessarily introduces a systematic uncertainty that predominantly affects the Milgrom fits, we have not been able to devise a stable method of determining more accurate values directly from the dynamics of the galaxies. Rotation curve data extending further into the flat portion of the rotation curve force a stable distance determination from Milgrom fits; this is clearly an important limitation of the data set considered here.

\subsection{Projection Angles}

Systematic uncertainties in projection angles substantially affect derived $\Upsilon$ values (see the discussion in $\S 3.1$ ). However, the estimates of systematic uncertainties in the projection angles appear to be reasonably behaved. Our sample contains 14 galaxies for which the uncertainty in $\Upsilon$ from the estimated systematic uncertainty in projection angles is less than twice the statistical uncertainty (see Table 1); in most of these cases the systematic error is less than the statistical error. A cursory examination of the model fits for these 14 galaxies shows that the various halo models or the Milgrom model are preferred as the best-fitting model in roughly the same fractions as for the full set of galaxies. More importantly, the galaxies of this subsample tend to have $|\lambda| \lesssim 10$, while the full sample contains several cases with $|\widetilde{\lambda}| \gtrsim 100$. These large values are likely a signal that the straightforward statistical comparison may be suspect, and that systematic errors arising from projection angles or other sources are contributing to the strong rejection of the Milgrom model.

\subsection{Nonaxisymmetry}

Many of the galaxies in the sample have bar and/or spiral features or lopsided shapes. In these cases, the assumption of axisymmetry is manifestly violated, although if the irregularities are weak (as is generally the case) the effect on the rotation curve will be small. Departures from regular elliptical (projected) isophotal shapes can indicate recent merging or tidal interactions which substantially distort the velocity field. These distortions are evident in maps of the differences between circular orbit models and kinematic data (e.g., see Figure 5 in Barnes \& Sellwood 2003). The effect of departures from axisymmetry requires painstaking modelling (e.g., Weiner et al. 2001), but incisive comparisons of halo models with Milgrom fits should be limited to galaxies with no gross departures from axisymmetry. Our sample contains roughly equal numbers of galaxies with strong (18) and weak (22) spiral structure. Seven galaxies have some evidence for a bar; 5 are strong bars in galaxies with strong spiral structure, and 2 are short/weak bars in galaxies with weak spiral structure. The specific galaxies discussed in the following section have only weak spiral structure.

\section{ESO 323G42 \& ESO 382G06}

MOND is vastly more falsifiable than dark matter hypotheses. Since the fitting parameters are few and understood more easily than the usual halo parameters, any one galaxy that can not be fit by the Milgrom relation could, in principle, refute the idea. Most galaxies in our sample have better halo fits than Milgrom fits; however, as discussed in $\S 5.1$, these relative likelihoods may be the result of systematic errors.

A better way to rule out MOND is simply to find galaxies with poor Milgrom fits in an absolute sense. This criterion also is hard to define as measures of fit quality are also affected by systematic errors. One simple way to judge the quality of a Milgrom fit is in a relative sense, by comparing with the Newtonian stars-only model. If the Milgrom relation provides an accurate description of galaxies, then Newtonian fits should never be better than Milgrom fits (they will be comparable if all of the measured velocities are close enough to the galaxy center that they are still in the Newtonian gravitational limit of the Milgrom force law). Many of the systematic effects that the halo fits can compensate for will affect both Milgrom fits and Newtonian fits equally, since both have little model freedom to compensate for systematic errors. It is difficult to see, for example, how an unaccounted-for distribution of gas could improve the fit of a Newtonian model relative to a Milgrom model; it should affect both equally and in the same direction relative to the measured velocities.

Finding galaxies whose Newtonian fits are clearly superior to Milgrom fits can kill MOND as a fundamental theory. (Note that galaxies whose Newtonian fits are superior to dark halo fits also pose severe problems for dark matter models.) Here we focus on two galaxies, ESO $323 \mathrm{~g} 42$ and ESO 382g06, whose Milgrom fits are worse than their Newtonian stars-only fits. Their 
rotation curves and several model curves are shown in Fig. 4(a) and (b), respectively.

In practice, the path to ruling out MOND is not so clear as systematic uncertainties complicate the situation. For these two galaxies, the residuals from our kinematic fitting do not show organized patterns associated with strong bars and/or spirals, and we believe these galaxies are quite axisymmetric. Any spiral structure present likely perturbs circular speeds by $<10 \mathrm{~km} / \mathrm{s}$, and the resulting small rotation curve features would be similar in both Newtonian and Milgrom fits. Without detailed information about the gas distribution, it is certainly possible that these Milgrom models are performing poorly simply because they have incorrect input. Further, the observed discrepancies between the Milgrom models and the data have magnitudes $(\leqslant 10 \%)$ that could possibly be lifted by including the gas (Broeils 1992; Verheijen 1997). We note that this is a question that can be addressed with further observations.

\subsection{ESO 323g42}

Figure 4(a) shows that the Milgrom relation fit (solid line) is clearly a poorer approximation to the data than either the Newtonian stars-only fit (dotted line) or the PI fit (dot-dashed line and the best halo fit) over the entire radial range of the data, $\approx 3.4$ disk scalelengths $R_{d}$ (the NFW curve has been omitted for clarity but is very similar to the PI curve). The systematic projection angle uncertainties for this disk are quite small $\left(\approx 2^{\circ}\right)$ and likely do not impact the fit strongly. Unfortunately, this galaxy is also one of the cases where no stable fit could be found when the galaxy distance is left as a free parameter. The Hubble distance may not be the correct value, but there is no reliable evidence to change it. The discrepancy between the Milgrom curve and the data is most evident where the model underpredicts $v_{c}$ near 5 kpc and overpredicts $v_{c}$ beyond $12 \mathrm{kpc}$. A reasonable gas distribution could rectify these discrepancies. However, one problem with adding significant amounts of gas to the model is that the model $\Upsilon_{D, I}$ value necessarily has to decrease. The $\Upsilon_{D, I}$ value in Table 1 is 0.78 , already somewhat lower than the average value. Looking at the PI model curve, it is apparent that departures from axisymmetry are quite small, $<5 \mathrm{~km} / \mathrm{s}$. Any mild spiral structure that may be present is not greatly influencing the model.

\subsection{ESO 382g06}

Unlike the case of ESO 323g42, the Milgrom fit for ESO 382g06 basically agrees with the both PI and Newtonian stars-only fits everywhere (out to the last point at $\approx 4.3 R_{d}$ ), except in the very central region of the galaxy. In that region, no model explains the data well. One possible explanation is that, despite lacking a clear signature in either the photometric or kinematic residuals, the center of ESO $382 \mathrm{~g} 06$ harbors some kind of non-axisymmetric structure. The rotation curve of ESO $382 \mathrm{~g} 06$ decreases slightly towards the edge of the optical disk, which is not so uncommon for late type spirals (Beauvais \& Bothun 2001). Such behavior in the low-acceleration regime causes the Milgrom relation fit to underperform the halo models, since it unavoidably gives asymptotically flat rotation curves. Note that this discrepancy is directly tied to the shape of the rotation curve, and will persist even including an undetected gas component. The projection angle uncertainties for this galaxy are about average for this sample (as determined in Barnes \& Sellwood 2003), $\approx 5^{\circ}$. However, it is unlikely that changing the projection angles will change the slope of the outer part of the rotation curve. Following the procedure described in $\S \mathbf{5}$, differing amounts of central pixels have been removed from the fit, and in three trials stable distance values have been found. Unfortunately, these values do not agree with each other very well and the fits give unphysically small amounts of mass to the disk. Consequently, we again find that changing the adopted distance to the galaxy will not improve the Milgrom fit.

\subsection{Variable $\Upsilon_{D, I}$ Fits}

In Section 5 , we have discussed some of the observational systematic uncertainties that could adversely influence the Milgrom fits. Here we consider the impact of a systematic modeling error due to the fundamental assumption of a constant mass-light ratio $\Upsilon$. Milgrom fits will be particularly susceptible to this type of error because of the deterministic dependence of the rotation curve on the baryonic mass. Halo models of any kind generally have sufficient freedom to contort themselves so that mild $\Upsilon$ gradients leave the quality of a halo fit unchanged.

We have performed Milgrom fits for three galaxies (ESO 322g76, ESO 323g42, and ESO 382g06) allowing the $\Upsilon_{D, I}$ values to vary radially; $\Upsilon_{B, I}$ values continue to be treated as spatially constant. Specifically, we assume a linear relation,

$$
\Upsilon_{D, I}(r)=\Upsilon_{D, 0}+m r,
$$

where $\Upsilon_{D, 0}$ is the central disk $\Upsilon$ value and $m$ is the slope of the relation in $\left(M_{\odot} / L_{\odot}\right) / \mathrm{kpc}$. The rotation curves for the variable $\Upsilon_{D, I}$ mass models are compared to the data points, the constant $\Upsilon$ curves, and the PI halo curves in Figure 5. The solid lines correspond to the variable $\Upsilon_{D, I}$ fits, the dot-dashed lines are the PI halo fits, and the dotted lines are the constant $\Upsilon$ fits.

The fit for ESO 322g76 (Figure 5a) serves as a test case since the constant $\Upsilon$ Milgrom fit is comparable or preferred to all the other models. We find $\Upsilon_{D, 0}=1.30$, $\Upsilon_{B, I}=1.75$, and $m=3.5 \times 10^{-3}$. Since the slope is small and the $\Upsilon$ values are nearly unchanged from their original values $\left(\Upsilon_{D, I}=1.33, \Upsilon_{B, I}=1.73\right)$, it is not surprising that the fits are equally good (for both fits, $\chi_{b}^{2}=2.05$ ). For this galaxy, with an acceptable Milgrom model with constant $\Upsilon$, the additional freedom does little to change the previous fit.

This is not the case for the two galaxies that appear to have problematic Milgrom fits. Allowing for $\Upsilon_{D, I}$ gradients, ESO $323 \mathrm{~g} 42$ (Figure 5b) is best fit with the parameters $\Upsilon_{D, 0}=\Upsilon_{B, I}=1.16, m=-4.0 \times 10^{-2}$. The resulting $\chi_{b}^{2}$ value is 1.54 , roughly $90 \%$ of the constant $\Upsilon_{D, I}$ value, making it comparable to the constant $\Upsilon$ halo and Newtonian fits. The change in $\Upsilon_{D, I}$ value from the center to the edge of the kinematic data is 0.29 , much larger than either uncertainty given in Table 1. This suggests that including the $\Upsilon$ gradient is truly improving the fit for this galaxy. The best-fit parameters for 
ESO 382g06 (Figure 囵) are $\Upsilon_{D, 0}=\Upsilon_{B, I}=3.81$ and $m=-2.5 \times 10^{-1}$. This case has a slight decrease in $\chi_{b}^{2}$ value, from 2.48 with constant $\Upsilon_{D, I}$ values to 2.46 . In this case, the $\Upsilon_{D, I}$ value changes from center to edge by 1.2 , smaller than just the statistical uncertainty alone in Table 1. While the quality of the fit has definitely improved, it is not as clear in this case that allowing a $\Upsilon$ gradient has significantly changed the model.

Qualitatively, the $\Upsilon_{D, I}$ slopes for these two galaxies act in the direction expected from observations of color gradients, with the outermost regions having lower $\Upsilon_{D, I}$ values than the centers (de Jong 1996). Multi-band photometry would allow determination of the actual color gradient, and whether this gradient is consistent with the best fit value.

ESO 323g42 and ESO 382g06 exemplify the difficulties associated with falsifying the Milgrom relation. While there are certainly hints of problems with the zerothorder models, plausible systematic effects (distance, gas mass, $\Upsilon$ gradients) must be exhausted before a definitive judgement can be handed down. For Milgrom fits with non-constant mass-light ratios, further observations can provide consistency checks, unlike halo models where all of our ignorance is folded into halo parameters and independent direct measurements of individual halo shapes are generally not possible.

\section{SUMMARY \& CONCLUSIONS}

We have investigated MOND mass models of 40 high surface surface brightness spiral galaxies. Using twodimensional Fabry-Perot velocity maps and $I$-band photometric images, best-fit bulge and disk $\Upsilon$ values have been determined using the Milgrom relation (Equation 11. As in our previous work using dark matter halo models (Barnes et al. 2004, BSK), we find that the systematic uncertainties due to projection angle uncertainties are typically much larger than the statistical uncertainties. It should be kept in mind that large systematic uncertainties could mask differences between various models and make any statistical comparison questionable.

We find that Milgrom model $\Upsilon_{D, I}$ values correlate with $B-R$ galaxy color (Figure 2 $\mathrm{b}$ ), in line with population synthesis models (Bell \& de Jong 2001; Bell et al. 2003). While the correlation does not exactly match the synthesis model trend and contains much scatter, it sharply contrasts with the results of BSK where no trend was found (Figure 2a). For a subsample of 8 galaxies that lie in the Hydra cluster, we find that they define a fairly tight $\Upsilon_{D, I^{-}}$-color correlation (Figure 3). Again, the correlation does not agree with the trends from population synthesis models in either slope or intercept. This contrasts with the findings in McGaugh (2004) which show excellent agreement between Milgrom model and population synthesis $\Upsilon$-color relations. We note that the galaxies used in McGaugh (2004) are the same galaxies used to calibrate the population synthesis prediction in Bell \& de Jong (2001). We feel confident that the disagreement we see can not stem from distance errors, since the mean of the distances to these galaxies is the same as an independent determination using the Fundamental Plane (Hudson et al. 1997). It could be argued that bulge contamination skews our results, but these are all late-type spirals with very little bulge. It is difficult to imagine that these bulges could cause such a radical shift in the correlation. Recognizing that the common view is that IMFs are universal, one possible explanation made plain by the model calculations of Bell \& de Jong (2001) is that there are different IMFs for these two sets of galaxies. With only 8 galaxies, the evidence is weak to challenge the standard view.

Utilizing a Bayesian model comparison technique (§ 4 and $\S \mathrm{A}$ ), we have compared Milgrom models to Newtonian stars-only, pseudo-isothermal halo, Navarro-FrenkWhite halo, and power law halo models. The results are presented in Table 2. Overall, halo models are statistically favored over Milgrom models. At the same time, Milgrom models are generally preferred to Newtonian stars-only models. Unfortunately, this kind of analysis takes statistical uncertainties only into account. The quality of the model fits is also influenced by systematic observational uncertainties (distance, inclination, etc.; see $\S$ (5). The Milgrom models are also subject to systematic modeling effects (like assuming constant massto-light ratios) that are more easily absorbed in halo models.

We have also discussed two specific galaxies which appear to present a problem for the Milgrom relation ( $(6)$. Our investigation suggests that nonaxisymmetry, projection angle uncertainties, or distance errors are not responsible for the Newtonian stars-only fits being better than the Milgrom fits. These galaxies provide excellent tests of the Milgrom relation since in this picture no galaxy should show Newtonian behavior in the lowacceleration (large radius) regime.

With an eye towards explaining away the seemingly Newtonian behavior in these two galaxies, we have performed additional fits allowing the $\Upsilon$ of the disk to vary linearly with radius. We find that for the galaxy ESO $322 \mathrm{~g} 76$, which is statistically well explained by the Milgrom relation, the best-fit parameters of a constant $\Upsilon_{D, I}$ are virtually unchanged by allowing $\Upsilon_{D, I}$ to vary. However, for the two problem galaxies ESO 323g42 and ESO $382 \mathrm{~g} 06$ we find that 1) the best-fit parameters of the varying $\Upsilon_{D, I}$ model are quite different than the constant $\Upsilon_{D, I}$ model; 2) the quality of the fits improve to the point of be comparable to halo fits; and 3 ) the predicted $\Upsilon_{D, I}$ variations agree qualitatively with observed variations. It is possible that a more quantitative match could be recovered with the proper observations.

In conclusion, while we find little statistical evidence to support the claim that Milgrom models explain the behavior of this sample of galaxies more economically than do dark matter models, there are several sources of systematic uncertainty that complicate the situation. Given these systematic uncertainties (distance, gas content, $\Upsilon$ gradients), it is difficult to see how Milgrom mass models are of lower quality than dark matter halo models. However, if one believes that stellar population models accurately describe galaxies, then correct models of the stellar components of real galaxies should reveal trends expected from such models. This is one piece of evidence in favor of Milgrom over dark matter models; Milgrom model $\Upsilon$ values show more of a trend with color than do dark matter model $\Upsilon$ values for the same galaxies.

Dynamical analyses which purport to rule out the Milgrom relation must be done on carefully selected galaxies for which systematic errors in dynamical assumptions are 
minimized. It is easy to demonstrate superior dynamical fits by dark matter halos than by the Milgrom relation, but on closer examination it is clear that in the majority of cases, the discrepancy can be explained by reasonable assumptions about particular known systematic errors.

The authors gratefully acknowledge the assistance and advice of Povilas Palunas, Ted Williams, Stacy McGaugh, and an anonymous referee. Ted Williams provided the imaging and Fabry-Perot data on which this paper is based. We also thank Tad Pryor, Eric Peng, Pat Côté, and Laura Ferrarese for several helpful discussions. This work was supported by NASA grant NAG510110, and AK has been partly supported by NSF grant AST-0546035 while finishing this work. We have used the NASA/IPAC Extragalactic Database (NED), which is operated by the Jet Propulsion Laboratory, California Institute of Technology, under contract with the National Aeronautics and Space Administration.

\section{APPENDIX}

\section{BAYESIAN STATISTICS PRIMER}

The application of Bayesian reasoning to statistical problems in astronomy has become much more widespread in recent years, and numerous textbook treatments exist. Here we give only a brief summary of Bayesian model comparison used in this paper, and point the interested reader to Jaynes (2003); Bretthorst (1990).

Bayesian statistics is based on Bayes' theorem,

$$
P(M \mid D I)=\frac{P(M \mid I) P(D \mid M I)}{P(D \mid I)} .
$$

$P(M \mid D I)$ is read as "the probability that $M$ is true given $D$ and $I$ ". Let us make this concrete. Say that proposition $M$ is a given model, proposition $D$ is the data fit by the model, and proposition $I$ is any initial information we have about the system. The left hand side of Equation A1 is then the probability that a model is true given the data and prior information. If we have one data set, two models, and the same prior information, we can write Equation A1 for each model $M_{j}$ and $M_{k}$. Since $D$ and $I$ are the same in both equations, we can divide the two to cancel $P(D \mid I)$. We now have a ratio of the probabilities of the models being correct,

$$
\frac{P\left(M_{j} \mid D I\right)}{P\left(M_{k} \mid D I\right)}=\frac{P\left(M_{j} \mid I\right) P\left(D \mid M_{j} I\right)}{P\left(M_{k} \mid I\right) P\left(D \mid M_{k} I\right)} .
$$

Following Jaynes (2003) we have that,

$$
\begin{aligned}
P(D \mid M I) & =\int d \boldsymbol{\Theta} P(D \mid \boldsymbol{\Theta} M I) P(\boldsymbol{\Theta} \mid M I) \\
& =\int d \boldsymbol{\Theta} L(\boldsymbol{\Theta}) P(\boldsymbol{\Theta} \mid M I),
\end{aligned}
$$

where $\boldsymbol{\Theta}$ are the $(1 \ldots N)$ free parameters, $L$ is the likelihood of a model, and $P(\boldsymbol{\Theta} \mid M I)$ is the prior probability. If we normalize the likelihood by its maximum value $L_{\max }$, we have that $P(D \mid M I)=L_{\max } W$, where $W$ is the Ockham factor,

$$
W \equiv \int d \Theta \frac{L(\Theta)}{L_{\max }} P(\Theta \mid M I) .
$$

In order to calculate the Ockham factor we need to decide what to use for the prior parameter probability. In the absence of any specific knowledge of the parameters, we choose a top-hat prior, i.e., a constant value $p$ in a specified range. Since the prior probability must be normalized, we can easily calculate what $p$ is. $\int P(\Theta \mid M I) d \Theta=1$ implies that $p=1 /\left(\Delta \Theta_{1} \Delta \Theta_{2} \ldots \Delta \Theta_{N}\right)$, where $\Delta \Theta_{j}$ is the range of a given free parameter. With this prior we can write,

$$
\begin{aligned}
P(D \mid M I) & =L_{\max } p \int d \Theta \frac{L(\boldsymbol{\Theta})}{L_{\max }} \\
& =L_{\max } p V(\Omega),
\end{aligned}
$$

where $V(\Omega)$ is just the integral of the normalized likelihood over all parameter space and is related to the volume of allowed parameter space. We are now ready to rewrite the right-hand side of Equation A2.

$$
\frac{P\left(M_{j} \mid D I\right)}{P\left(M_{k} \mid D I\right)}=\frac{P\left(M_{j} \mid I\right)}{P\left(M_{k} \mid I\right)} \frac{L_{\max , j}}{L_{\max , k}} \frac{p_{j}}{p_{k}} \frac{V_{j}\left(\Omega_{j}\right)}{V_{k}\left(\Omega_{k}\right)} .
$$

Assuming our prior information leaves us equally ignorant about both models, the first term on the right-hand side of Equation A6] is 1. We are left with an expression that is simple and sensible. If $M_{j}$ provides a better fit (i.e., has a smaller $\chi_{b}^{2}$ ) than $M_{k}$, then the likelihood ratio (the second term) is large, thus pushing the ratio in favor of $M_{j}$, as one would expect. If the two models have the same free parameters, then the third ratio relating the parameter ranges is 1 and the last ratio is $\approx 1$. We are left with the obvious answer that the model with the highest likelihood is the more probable model. However, the real power of this approach emerges when you compare models with different 
numbers of parameters. For concreteness, let us say that $M_{j}$ has two free parameters $\alpha$ and $\beta$ and model $M_{k}$ has two additional parameters $\gamma$ and $\delta$. We know $L_{\max }$ for each model, and we assume that $L_{\max , k}<L_{\max , j}$. We also know that, $p_{j}=1 /(\Delta \alpha \Delta \beta)$ and $p_{k}=1 /(\Delta \alpha \Delta \beta \Delta \gamma \Delta \delta)$, so the $p$ ratio term is $\Delta \gamma \Delta \delta$. Since $M_{k}$ has the larger number of parameters, let us suppose that $V_{j}\left(\Omega_{j}\right)>V_{k}\left(\Omega_{k}\right)$. The likelihood ratio is $<1$, the $V$ ratio is $>1$, and the $p$ ratio is dependent on the ranges that we believe two of the parameters fall in. The Bayesian model probability ratio does not necessarily choose the model with the highest likelihood: it determines if the benefit of adding more fitting parameters balances the cost of making the model more complicated.

\section{REFERENCES}

Anderson, K. S. J., Baggett, S. M., \& Baggett, W. E. 2004, AJ, 127,2085

Angus, G. W., Shan, H., Zhao, H., Famaey, B. astro-ph/0609125

Barnes, E. I., Sellwood, J. A. 2003, AJ, 125, 1164

Barnes, E. I., Sellwood, J. A., Kosowsky, A. 2004, AJ, 128, 2724

Beauvais, C., Bothun, G. 2001, ApJS, 136, 41

Begeman, K. G., Broeils, A. H., Sanders, R. H. 1991, MNRAS, 249,523

Bekenstein, J., Milgrom, M. 1984, ApJ, 286, 7

Bekenstein, J. D. 2004, astro-ph/0403694

Bell, E. F., de Jong, R. S. 2001, ApJ, 550, 212

Bell, E. F., McIntosh, D. H., Katz, N., Weinberg, M. D. 2003, ApJS, 149,289

Blais-Ouellette, S., Amram, P. \& Carignan, C. 2001, AJ, 121, 1952

Bothun, G. D., Schommer, R. A., Williams, T. B., Mould, J. R., Huchra, J. P. 1992, ApJ, 388, 253

Bradac, M., Clowe, D., Gonzalez, A. H., Marshall, P., Forman, W., Jones, C., Markevitch, M., Randall, S. W., Schrabback, T., Zaritsky, D. 2006, astro-ph/0608408

Bretthorst, G. L. 1990, in Maximum Entropy and Bayesian Methods, ed. P. F. Foug'ere (Dordrect:Kluwer), 53

Broeils, A. H. 1992, Ph.D. Thesis, Groningen University

Catinella, B., Giovanelli, R., Haynes, M. 2006, ApJ, 640, 751

Christensen, N., Meyer, R., Knox, L., Luey, B. 2001, Classical and Quantum Gravity, 18, 2677

Clowe, D., Bradac, M., Gonzalez, A. H., Markevitch, M., Randall, S. W., Jones, C., Zaritsky, D. 2006, astro-ph/0608407

Courteau, S., Rix, H.-W. 1999, ApJ, 513, 561

Courteau, S. 2003, Rev. Mexicana Astron. Astrofis., 17, 171

de Blok, W. J. G., McGaugh, S. S. 1998, ApJ, 508, 132

de Jong, R. S. 1996, A\&A, 313, 377

Gentile, G., Salucci, P., Klein, U., Vergani, D., Kalberla, P. 2004, MNRAS, 351, 903

Hudson, M. J., Lucey, J. R., Smith, R. J., Steel, J. 1997, MNRAS, 291488

Jaynes, E., ed. Bretthorst, G. L. 2003, Probability Theory : The Logic of Science, Cambridge Univ. Press, Cambridge

Kent, S. M. 1987, AJ, 93, 816

Kosowsky, A., Milosavljević, M. \& Jimenez, R. 2002, Phys. Rev. D, 66, 063007
Krumm, N., Salpeter, E. E. 1979, AJ, 84, 1138

Lake, G. \& Feinswog, L. 1989, AJ, 98, 166

Liddle, A. R. 2004, MNRAS, 351, 49

McGaugh, S. S., Schombert, J. M., Bothun, G. D., de Blok, W. J.

G. 2000, ApJ, 533L, 99

McGaugh, S. S. 2004, ApJ, 609, 652

McGaugh, S. S. 2005, ApJ, 632, 859

Milgrom, M. 1983, ApJ, 270, 365

Milgrom, M. 1983, ApJ, 270, 371

Milgrom, M. 1983, ApJ, 270, 384

Navarro, J. F., Frenk, C. S., White, S. D. M. 1996, ApJ, 462, 563

Palunas, P., Williams, T. B. 2000, AJ, 120, 2884 (PW)

Persic, M., Salucci, P., Stel, F. 1996, MNRAS, 281, 27

Press, W. H., Flannery, B. P., Teukolsky, S. A., Vetterling, T. A. 1992, Numerical Recipes (Cambridge:Cambridge Univ. Press)

Rohlfs, K., Boehme, R., Chini, R.k Wink, J. E. 1986, A\&A, 158, 181

Rubin, V. C. 1979, Comments on Astrophysics, 8, 79

Salpeter, E. E. 1978, IAUS, 77, 23

Sancisi, R., Allen, R. J. 1979, A\&A, 74, 73

Sancisi, R., Allen, R. J., Sullivan, W. T., III 1979, A\&A, 78, 217

Sanders, R. H. 1996, ApJ, 473, 117

Sanders, R. H., Verheijen, M. A. W. 1998, ApJ, 503, 97

Sanders, R. H., McGaugh, S. S. 2002, ARA\&A, 40, 263

Schlegel, D. J., Finkbeiner, D. P., Davis, M. 1998, ApJ, 500,525

Sprayberry, D., Bernstein, G. M., Impey, C. D., Bothun, G. D. 1995, ApJ, 438, 72

Tully, R. B., Fisher, J. R. 1977, A\&A, 54, 661

Tully, R. B., Pierce, M. J., Huang, J., Saunders, W., Verheijen, M. A. W., Witchalls, P. L. 1998, AJ, 115, 2264

van Albada, T. S., Bahcall, J. N., Begeman, K., Sancisi, R. 1985, ApJ, 295, 305

van Moorsel, G. A. 1982, A\&A, 107, 66

Verheijen, M. A. W. 1997, PhD Thesis, University of Groningen

Weiner, B.J., Sellwood, J.A., and Williams, T.B. 2001, ApJ, 546, 931

Zwaan, M. A., van der Hulst, J. M., de Blok, W. J. G., McGaugh, S. S. 1995, MNRAS, 273, 35 
TABLE 1

Best-Fit $\chi_{b}^{2}$ VAlues, $\Upsilon$ values (With Statistical uncertainties $\sigma$ ), AND Systematic uncertainties $(s)$ For Milgrom models. The last COlumn CONTAINS THE RATIO BETWEEN THE KINEMATIC DATA'S RADIAL EXTENT $R_{\mathrm{k}, \max }$ AND THE DISK SCALELENGTH $r_{d}$.

\begin{tabular}{|c|c|c|c|c|c|c|}
\hline Galaxy & $\chi_{b}^{2}$ & $\Upsilon_{D, I_{-\sigma}^{+\sigma}}^{+a}$ & $s \Upsilon_{D, I}$ & $\Upsilon_{B, I}^{+\sigma}$ & $s \Upsilon_{B, I}$ & $R_{\mathrm{k}, \max } / r_{d}$ \\
\hline ESO 215g39 & 1.48 & $1.28 \stackrel{0.07}{0.09}$ & 0.63 & $2.79_{0.25}^{0.32}$ & 0.57 & 3.35 \\
\hline ESO 216g20 & 4.16 & $2.08 \begin{array}{c}0.89 \\
0.07\end{array}$ & 0.14 & 1.310 .30 & 0.37 & 5.20 \\
\hline ESO $263 \mathrm{~g} 14$ & 1.93 & $0.81 \frac{0}{0.01}$ & 0.08 & & $\ldots$ & 4.64 \\
\hline ESO 267g29 & 5.42 & $2.14_{0.04}^{0: 04}$ & 1.77 & $2.14_{0.04}^{0.04}$ & 1.90 & 3.81 \\
\hline ESO 268g37 & 4.11 & $1.40 \begin{array}{l}0.03 \\
0.03\end{array}$ & 0.64 & & $\ldots$ & 3.78 \\
\hline ESO 268g44 & 2.05 & $1.200: 87$ & 0.08 & 3.090 .27 & 0.15 & 4.45 \\
\hline ESO $317 \mathrm{~g} 41$ & 4.58 & $2.08 \begin{array}{c}0.04 \\
0.04\end{array}$ & 0.04 & $1.43_{0.10}^{0.34}$ & 0.04 & 5.09 \\
\hline ESO 322g36 & 4.53 & $0.94_{0.03}^{8: 82}$ & 0.47 & 1.09 8:09 & 0.38 & 3.71 \\
\hline ESO $322 \mathrm{~g} 42$ & 3.67 & $0.48 \begin{array}{l}0.03 \\
0.02\end{array}$ & 0.02 & & $\ldots$ & 3.66 \\
\hline ESO 322g44 & 1.61 & $0.71_{0.03}^{0.03}$ & 0.06 & 0.721 .19 & 2.33 & 4.05 \\
\hline ESO $322 \mathrm{~g} 45$ & 2.16 & $1.43 \begin{array}{l}0.04 \\
0.04\end{array}$ & 0.02 & $1.43 \begin{array}{l}0.02 \\
0.04\end{array}$ & 0.02 & 2.11 \\
\hline ESO $322 \mathrm{~g} 76$ & 2.05 & $1.33_{0.0}^{8: 0}$ & 0.27 & $1.73_{0.15}^{0.15}$ & 0.19 & 4.47 \\
\hline ESO 322g77 & 3.25 & $3.110: 1$ & 0.13 & $2.080: 51$ & 0.23 & 6.33 \\
\hline ESO $322 \mathrm{~g} 82$ & 4.53 & $1.34 \begin{array}{l}0.06 \\
0.05\end{array}$ & 0.17 & $7.99 \begin{array}{l}0.38 \\
0.44\end{array}$ & 1.72 & 4.12 \\
\hline ESO 323g 25 & 2.81 & $\begin{array}{l}0.05 \\
0.4103 \\
0.03\end{array}$ & 0.83 & $\begin{array}{l}15.56 \frac{1.49}{1.26} \\
1.49\end{array}$ & 8.63 & 4.75 \\
\hline ESO 323g27 & 2.83 & 1.440 .07 & 0.13 & 5.100 .260 & 0.05 & 4.25 \\
\hline ESO 323g39 & 4.55 & $0.51 \begin{array}{l}0.03 \\
0.04\end{array}$ & 0.84 & & $\ldots$ & 3.09 \\
\hline ESO $323 \mathrm{~g} 42$ & 1.66 & $0.78 \begin{array}{l}0.84 \\
0.03\end{array}$ & 0.01 & $1.60_{0.21}^{0.28}$ & 0.08 & 4.50 \\
\hline ESO 323g73 & 3.10 & 1.29 & 0.29 & $\ldots$ & $\ldots$ & 5.11 \\
\hline ESO 374g03 & 3.29 & $0.69 \begin{array}{l}0.01 \\
0.02\end{array}$ & 0.04 & . & $\ldots$ & 3.84 \\
\hline ESO $375 \mathrm{~g} 02$ & 2.08 & $1.60 \begin{array}{l}0.05 \\
0.06\end{array}$ & 0.24 & $1.64_{0.36}^{0.32}$ & 0.43 & 5.35 \\
\hline ESO 381g05 & 2.75 & 5.460 .07 & 2.86 & & $\ldots$ & 3.73 \\
\hline ESO 382g06 & 2.48 & $3.07 \begin{array}{l}0.65 \\
0.86\end{array}$ & 1.11 & $3.42 \underset{0.47}{0.61}$ & 0.72 & 4.03 \\
\hline ESO $435 \mathrm{~g} 26$ & 11.98 & $1.79 \begin{array}{l}0.86 \\
0.03\end{array}$ & 1.05 & $3.20 \begin{array}{l}0.54 \\
0.48\end{array}$ & 1.38 & 6.04 \\
\hline ESO $437 \mathrm{~g} 04$ & 2.91 & 2.420 .03 & 0.23 & $2.42 \begin{array}{l}0.403 \\
0.02\end{array}$ & 0.23 & 4.51 \\
\hline ESO 437g31 & 1.07 & $2.07 \begin{array}{l}0.04 \\
0.04\end{array}$ & 1.49 & $2.07 \begin{array}{l}0.04 \\
0.04\end{array}$ & 1.65 & 3.74 \\
\hline ESO $438 g 08$ & 2.32 & 0.520 .05 & 0.29 & $3.66_{0.33}^{0.04}$ & 1.53 & 4.71 \\
\hline ESO $438 \mathrm{~g} 15$ & 2.60 & $0.920: 83$ & 0.10 & $1.66^{0}: 87$ & 0.09 & 6.62 \\
\hline ESO $439 \mathrm{~g} 18$ & 4.14 & $2.20_{0: 03}^{8: 82}$ & 0.37 & $2.20_{0: 02}^{8: 02}$ & 0.37 & 4.46 \\
\hline ESO 439g20 & 1.86 & $2.908: 8$ & 0.17 & $2.90_{0.03}^{0.03}$ & 0.42 & 3.98 \\
\hline ESO $444 \mathrm{~g} 47$ & 1.69 & $2.00 \begin{array}{l}0: 83 \\
0.05\end{array}$ & 0.15 & $2.00_{0.05}^{8: 83}$ & 0.15 & 4.95 \\
\hline ESO $445 \mathrm{~g} 15$ & 3.03 & $2.91_{0.18}^{0.06}$ & 0.17 & $1.58 \begin{array}{l}0.19 \\
0.17\end{array}$ & 0.13 & 5.17 \\
\hline ESO $445 \mathrm{~g} 19$ & 2.31 & 1.850 .04 & 0.03 & $1.12_{0.31}^{0.136}$ & 0.08 & 3.69 \\
\hline ESO 445g39 & 4.22 & $5.440: 37$ & 0.63 & $2.890: 40$ & 0.76 & 4.11 \\
\hline ESO $446 g 01$ & 2.23 & $1.50 \begin{array}{l}0.41 \\
0.02\end{array}$ & 0.50 & & $\ldots$ & 3.97 \\
\hline ESO 501g01 & 2.33 & $1.32 \quad 0.03$ & 0.44 & $1.32 \underset{0.04}{0.03}$ & 0.44 & 2.76 \\
\hline ESO 501g68 & 2.13 & 2.600 .22 & 0.31 & 4.760 .21 & 0.15 & 3.84 \\
\hline ESO 502g02 & 1.74 & 2.080 .02 & 0.02 & $2.080: 02$ & 0.02 & 4.50 \\
\hline ESO 509g80 & 3.75 & $2.76_{0.05}^{0.02}$ & 0.19 & 3.170 .17 & 0.14 & 4.83 \\
\hline ESO $569 \mathrm{~g} 17$ & 1.67 & $2.20 \begin{array}{l}0.03 \\
0.03\end{array}$ & 1.83 & & $\ldots$ & 5.72 \\
\hline
\end{tabular}

a $\Upsilon$ values have units $\left(\mathrm{M}_{\odot} / \mathrm{L}_{\odot}\right)$. 
TABLE 2

LOGARITHMS OF PROBABILITY RATIOS FOR THE VARIOUS MODElS. ThE COLUMNS LIST THE RELATIVE PROBABILITIES OF THE THE VARIOUS MODELS TO MILGROM MODELS. Negative values indicate that the Milgrom Model is PREFERRED. The LARGEST POSITIVE VALUE FOR A GALAXY COMES FROM THE MODEL (OTHER THAN MILGROM) THAT BEST DESCRIBES THE DATA.

\begin{tabular}{ccccc}
\hline \hline Galaxy & $\lambda_{\text {stars }- \text { only }}$ & $\lambda_{\text {PI }}$ & $\lambda_{\text {NFW }}$ & $\lambda_{\text {power-law }}$ \\
\hline ESO 215g39 & -44.66 & 40.26 & 32.27 & 23.78 \\
ESO 216g20 & -32.43 & 9.06 & 3.92 & 5.61 \\
ESO 263g14 & -49.46 & 7.16 & 4.38 & 0.29 \\
ESO 267g29 & -47.16 & 27.47 & 24.76 & 25.42 \\
ESO 268g37 & -170.16 & 33.57 & 17.81 & 1.97 \\
ESO 268g44 & 3.21 & 8.95 & 6.34 & 0.45 \\
ESO 317g41 & 0.05 & 4.24 & -15.56 & -17.21 \\
ESO 322g36 & -144.46 & 16.31 & 15.70 & 14.20 \\
ESO 322g42 & -81.50 & 49.17 & 11.56 & 12.90 \\
ESO 322g44 & -153.87 & 7.76 & 3.93 & 5.06 \\
ESO 322g45 & -39.33 & 14.59 & 11.65 & 13.18 \\
ESO 322g76 & -17.42 & 0.13 & -4.39 & -1.50 \\
ESO 322g77 & -3.96 & 8.18 & 7.07 & 8.02 \\
ESO 322g82 & 158.84 & 162.29 & 163.86 & 158.06 \\
ESO 323g25 & 59.15 & 177.19 & 195.25 & 113.44 \\
ESO 323g27 & -4.76 & 21.32 & 17.77 & 12.86 \\
ESO 323g39 & -55.35 & 20.97 & 12.84 & 11.75 \\
ESO 323g42 & 36.22 & 79.43 & 64.07 & 61.33 \\
ESO 323g73 & -94.97 & 53.64 & 51.04 & 52.33 \\
ESO 374g03 & -78.04 & 71.89 & 30.20 & 0.59 \\
ESO 375g02 & -84.92 & 21.97 & 21.95 & 20.80 \\
ESO 381g05 & -128.24 & 215.20 & 216.79 & 212.01 \\
ESO 382g06 & 10.70 & 9.89 & 6.46 & 7.60 \\
ESO 435g26 & 126.10 & 182.98 & 192.48 & 126.17 \\
ESO 437g04 & -153.84 & 207.50 & 171.75 & 158.56 \\
ESO 437g31 & -67.01 & 13.52 & -3.45 & -2.09 \\
ESO 438g08 & 77.78 & 76.00 & 73.35 & 74.73 \\
ESO 438g15 & -92.73 & 43.05 & 39.63 & 41.19 \\
ESO 439g18 & -114.20 & 73.18 & 63.88 & 35.15 \\
ESO 439g20 & -65.38 & 35.96 & 24.74 & 3.19 \\
ESO 444g47 & -96.68 & 14.98 & 2.95 & 5.09 \\
ESO 445g15 & -79.48 & -9.18 & -13.31 & -13.43 \\
ESO 445g19 & -67.36 & 7.28 & 13.19 & 13.93 \\
ESO 445g39 & -0.26 & 7.52 & 0.03 & 1.37 \\
ESO 446g01 & -219.48 & 4.36 & 0.25 & 1.24 \\
ESO 501g01 & -66.08 & 31.50 & 8.88 & -11.19 \\
ESO 501g68 & -4.56 & -1.74 & -4.70 & -2.35 \\
ESO 502g02 & -80.73 & 16.47 & 12.31 & 5.60 \\
ESO 509g80 & 56.56 & 169.81 & 166.45 & 90.89 \\
ESO 569g17 & 5.67 & 43.13 & 46.70 & 9.39 \\
& & & & \\
\hline & & & & \\
\hline
\end{tabular}



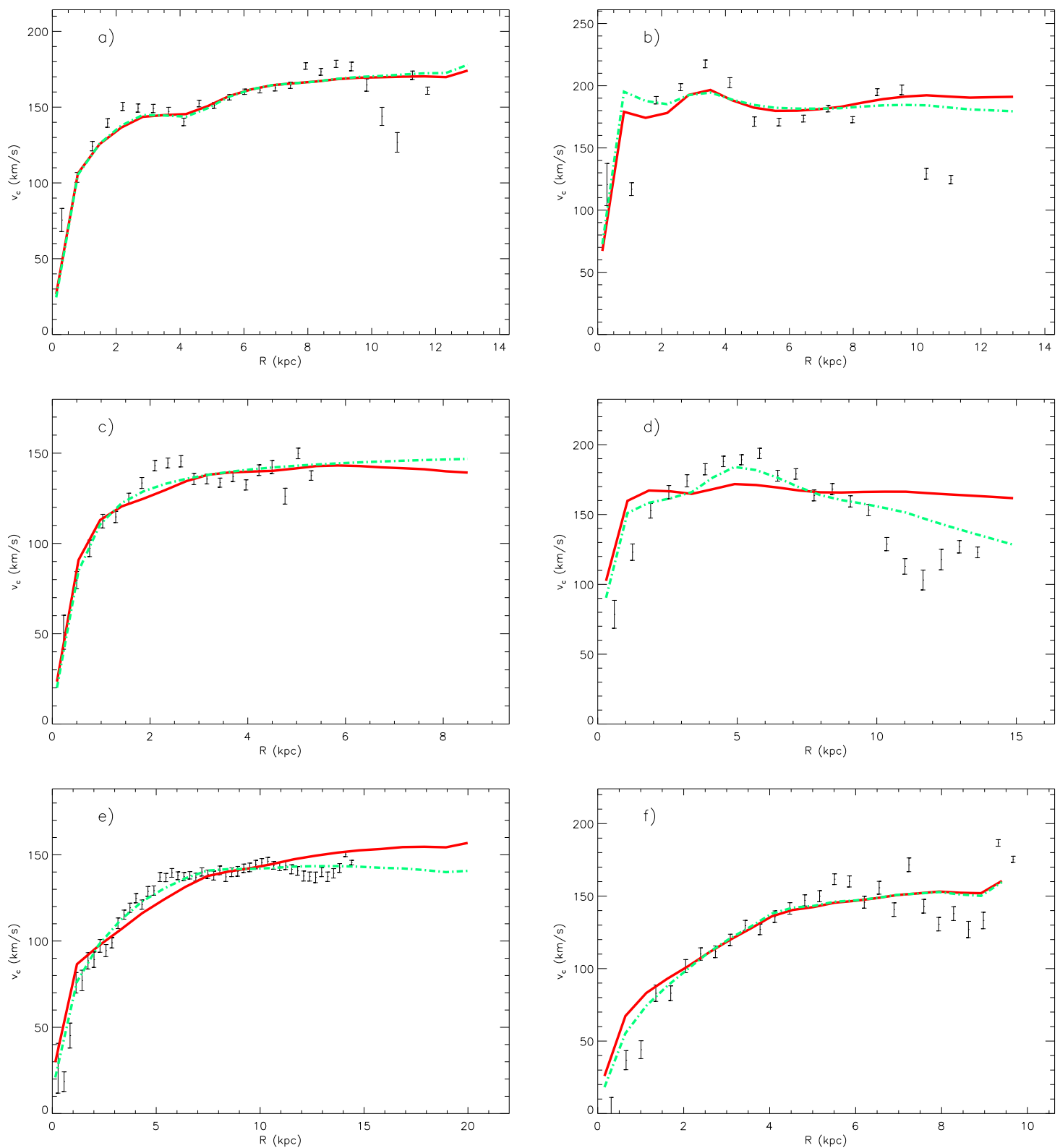

FIG. 1. - Comparisons between Milgrom relation and pseudo-isothermal halo rotation curves. The solid lines are the Milgrom rotation curves and the dot-dashed lines are the pseudo-isothermal halo curves. The error bars mark the circular speeds derived from the velocity map during kinematic fitting (Barnes \& Sellwood 2003). Panels a) (ESO 322g76) and b) (ESO 445g15) illustrate cases in which the Milgrom fit is preferred over or comparable to the other models. Panels c) (ESO 268g44) and d) (ESO 438g08) show two cases where the Milgrom models are not favored over any of the other models. ESO $268 \mathrm{~g} 44$ has substantial spiral structure and ESO $438 \mathrm{~g} 08$ appears to have an interacting companion. Panels e) (ESO 323g42) and f) (ESO 382g06) are the axisymmetric galaxies for which the Milgrom fit is never favored. 

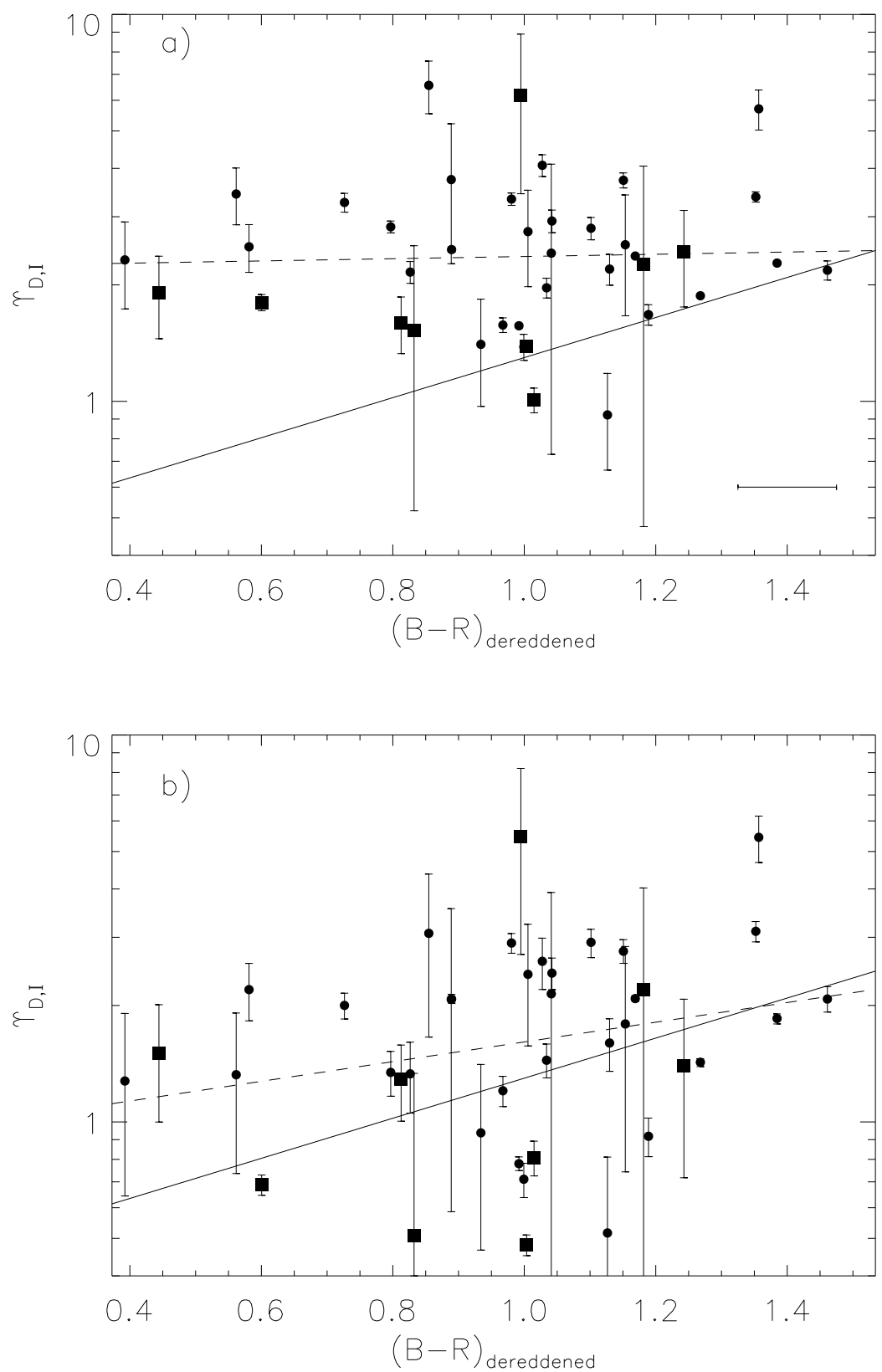

FIG. 2. - The color- $\Upsilon_{D, I}$ correlations for the galaxies in this sample assuming Newtonian stars-only models (a) and Milgrom models (b). The dashed line is the best linear fit to the points and the solid line is the fit from Bell et al. (2003). The error bar is the estimated uncertainty in galaxy color. 


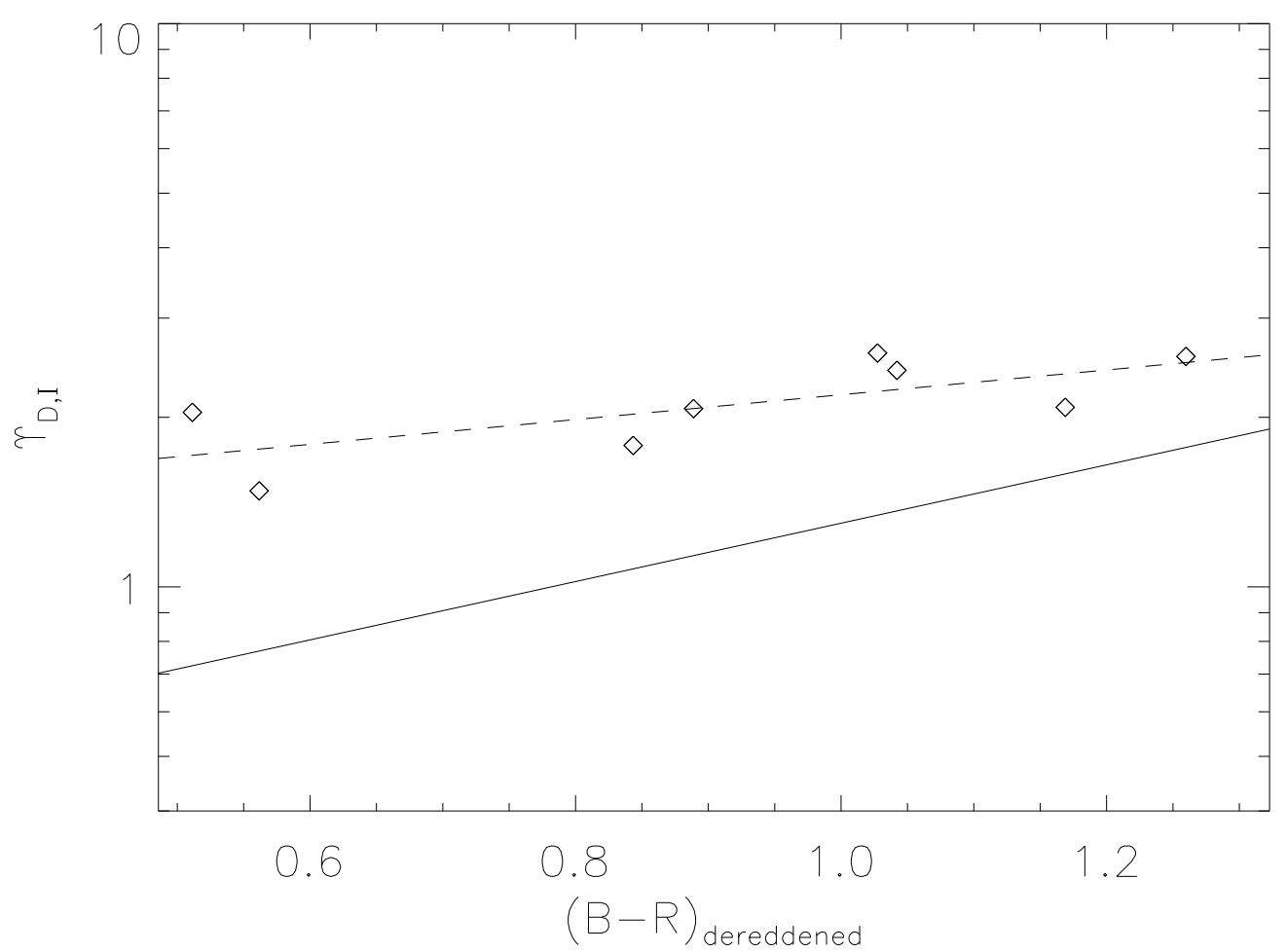

FIG. 3. - The color- $\Upsilon_{D, I}$ correlations for the Hydra cluster galaxies. Again, the dashed line is the best linear fit to the points and the solid line is the Bell et al. (2003) fit. 

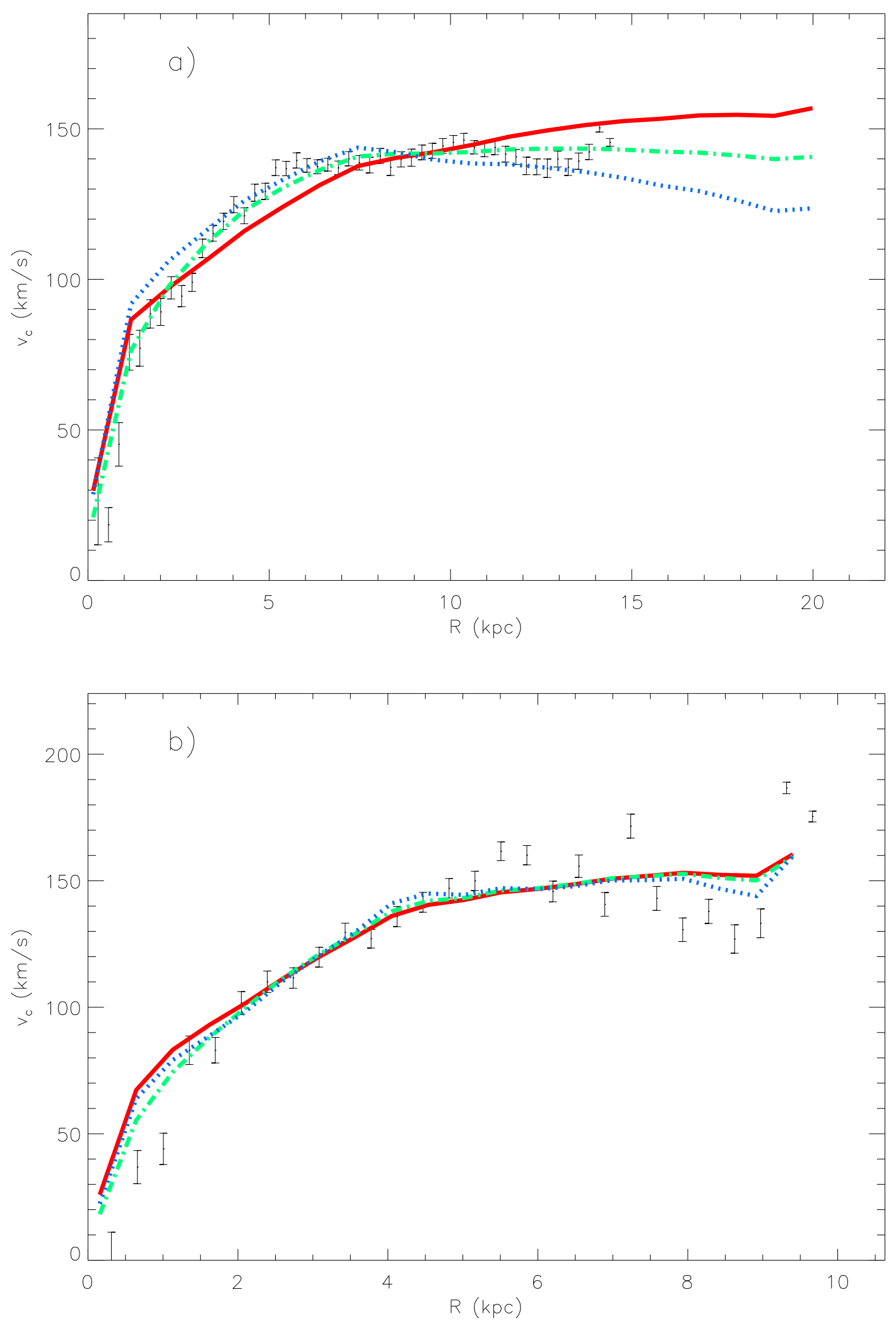

FIG. 4.- Comparisons between Milgrom (solid), pseudo-isothermal (dot-dashed), and Newtonian stars-only (dotted) rotation curves for ESO 323g42 (a) and ESO 382g06 (b). The points with error bars show the circular speeds derived from the velocity map during kinematic fitting (Barnes \& Sellwood 2003). 

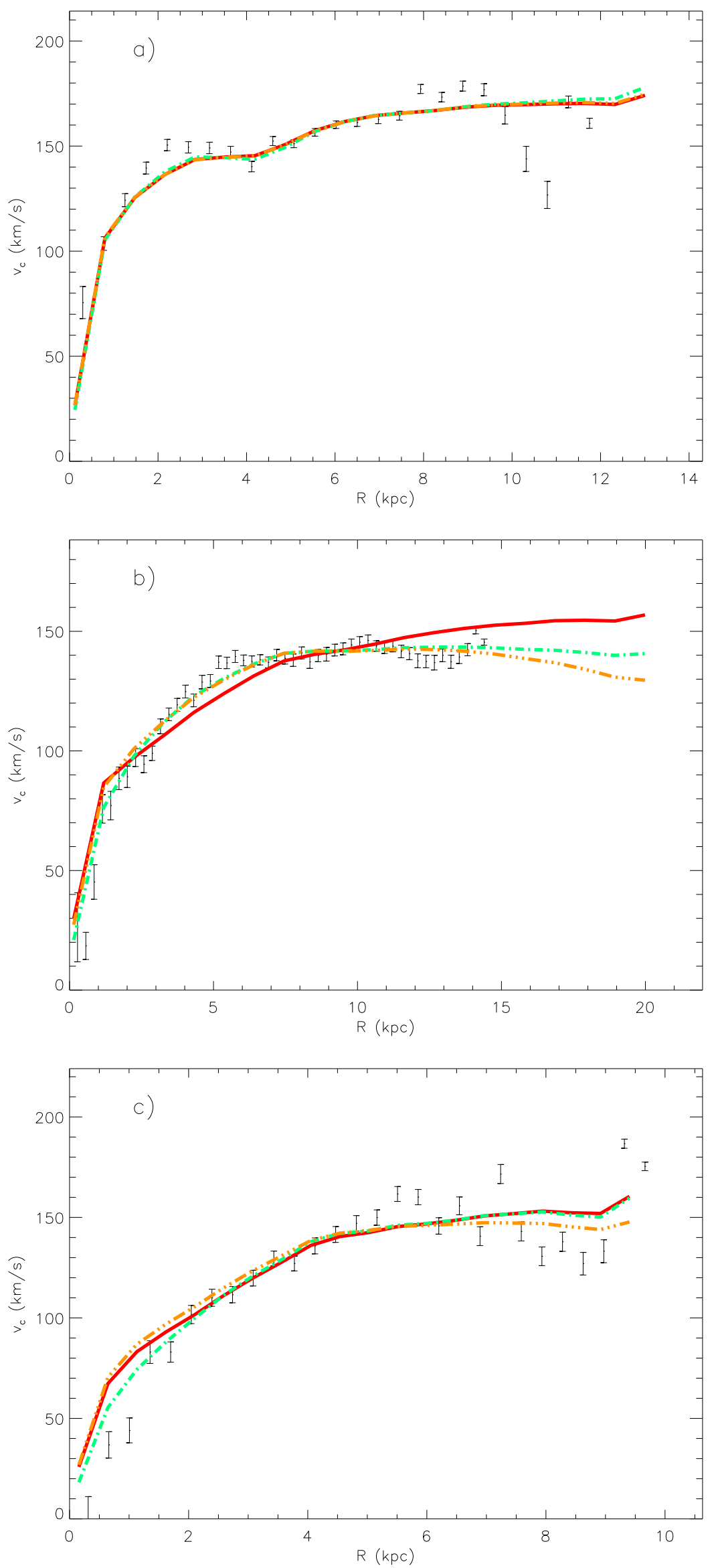

FIG. 5.- Comparisons between spatially-constant $\Upsilon_{D, I}$ Milgrom (solid lines), pseudo-isothermal (dot-dashed lines), and variable $\Upsilon_{D, I}$ Milgrom (triple-dot-dashed lines) rotation curves for ESO $322 \mathrm{~g} 76$ (a), 323g42 (b), and ESO $382 \mathrm{~g} 06$ (c). Again, the points with error bars denote the circular speeds derived from kinematic fitting of the velocity map. 